Portland State University

PDXScholar

Civil and Environmental Engineering Faculty

Publications and Presentations

Civil and Environmental Engineering

$10-2017$

\title{
Eulerian-Based Virtual Visual Sensors to Measure Dynamic Displacements of Structures
}

\author{
Ali Shariati \\ University of Delaware \\ Thomas Schumacher \\ Portland State University, thomas.schumacher@pdx.edu
}

Follow this and additional works at: https://pdxscholar.library.pdx.edu/cengin_fac

Part of the Civil and Environmental Engineering Commons

Let us know how access to this document benefits you.

\section{Citation Details}

Shariati, Ali and Schumacher, Thomas, "Eulerian-Based Virtual Visual Sensors to Measure Dynamic Displacements of Structures" (2017). Civil and Environmental Engineering Faculty Publications and Presentations. 425.

https://pdxscholar.library.pdx.edu/cengin_fac/425

This Post-Print is brought to you for free and open access. It has been accepted for inclusion in Civil and Environmental Engineering Faculty Publications and Presentations by an authorized administrator of PDXScholar. Please contact us if we can make this document more accessible: pdxscholar@pdx.edu. 


\section{Eulerian-Based Virtual Visual Sensors to Measure Dynamic}

2 Displacements of Structures

3

$4 \quad$ Ali Shariati ${ }^{1 * *}$ and Thomas Schumacher ${ }^{2}$

$5{ }^{1}$ Civil and Environmental Engineering, University of Delaware, Newark, DE 19716, USA; E-

$6 \quad$ mail: $\underline{\text { alish@udel.edu }}$

$8{ }^{2}$ Civil and Environmental Engineering, Portland State University, Portland, OR 97201, USA; E-

9 mail: thomas.schumacher@pdx.edu

10

11 * Author to whom correspondence should be addressed; E-Mail: alish@udel.edu;

12 Tel.: +1-443-449-1414; Fax: +1-302-831-3640.

14 Abstract: Vibration measurements provide useful information about a structural system's

15 dynamic characteristics and are used in many fields of science and engineering. Here, we present

16 an alternative non-contact approach to measure dynamic displacements of structural systems using

17 digital videos. The concept is that intensity measured at a pixel with a fixed (or Eulerian)

18 coordinate in a digital video can be regarded as a virtual visual sensor (VVS). The pixels in the

19 vicinity of the boundary of a vibrating structural element contain useful frequency information,

20 which we have been able to demonstrate in earlier studies. Our ultimate goal, however, is to be

21 able to compute dynamic displacements, i.e. actual displacement amplitudes in the time domain.

22 In order to achieve that we introduce the use of simple black-and-white targets (BWT) that are 
23 mounted to locations of interest on the structure. By using these targets, intensity can be directly

24 related to displacement, turning a video camera into a simple, computationally inexpensive, and

25 accurate displacement sensor with notably low signal-to-noise ratio (SNR). We show that subpixel

26 accuracy with levels comparable to computationally-expensive block matching algorithms can be

27 achieved using the proposed targets. Our methodology can be used for laboratory experiments, on

28 real structures, and additionally we see educational opportunities in the K-12 classroom. In this

29 paper we introduce the concept and theory of the proposed methodology, present and discuss a

30 laboratory experiment to evaluate the accuracy of the proposed BWT target, and discuss the results

31 from a field test of an in-service bridge.

33 Keywords: Vibration; Dynamic displacement; Structural health monitoring; Digital video; Virtual

34 visual sensor; Eulerian coordinate; Black-and-white target; Subpixel accuracy.

\section{Introduction}

37 Structural vibrations contain important information about a structural system's dynamic

38 characteristics. Changes over time in the vibration response can be caused by alterations in the

39 loading, boundary conditions, or degradation of the structural system. As such, structural health

40 monitoring (SHM) has emerged as a modern asset management support tool to help owners and

41 managers make more informed decisions regarding repair, optimal intervention, and management

42 of lifeline assets such as bridges during regular service and after extreme events such as natural

43 disasters. Vibration-based SHM methods use dynamic characteristics such as natural frequencies

44 and mode shapes to detect the occurrence of damage and estimate its location and severity [1]-[3].

45 A critical step in this process is the gathering of the vibration data using sensors or sensor networks. 
46 The ultimate goal is to have a sensing system that produces objective, quantitative, and accurate

47 data, inexpensively. Conventional contact-type sensors such as strain gages or accelerometers that

48 are attached to specific locations of a structure are capable of measuring the response at that

49 specific point. Accessibility of the member of interest combined with wiring issues in addition to

50 high local-only sensitivity are some drawbacks of conventional sensors, which have urged

51 innovation to develop non-contact sensors. On the other hand, laser interferometry instruments are

52 reliable but comparatively expensive as they use sophisticated equipment and require specialized

53 trained operators [4]. Photogrammetry methods have been used in the measurement of static

54 displacements and strains in bridges. More recently, digital image correlation (DIC) and other

55 block matching algorithms [5],[6] that use digital video data to measure static displacement fields

56 with high accuracy have been explored [7]-[10]. However, the computational cost of these

57 methods is relatively high. Efficient yet accurate non-contact methods are needed that are

58 computationally inexpensive and work with standard digital video cameras.

In this paper, we propose a simple alternative way to measure structural vibrations using

61 Eulerian-based virtual visual sensors (VVS), for which the fundamental basis we have developed

62 earlier [11]-[13] We show that for a black-and-white target (BWT), a linear intensity-displacement

63 relationship exists for a patch of pixels on the boundary of the target. We refer to this as intensity-

64 to-displacement transform (IDT). It should be noted that this same transform can also be applied

65 without using BTWs, for example to the edge of a structural member where a distinct boundary

66 between the member and the background exists. Fig. 1 illustrates the concept of our proposed

67 methodology: a BWT target is attached to a location of interest. From the digital video extracted

68 from the camera, a VVS located on the BWT is selected. The change of intensity is recorded over 
69 time (green curve) and by employing a linear transformation (= IDT) converted to actual

70 displacement (blue curve). Also shown is a frequency analysis (red curve), which can be obtained

71 directly from the intensity time history by using a Fast Fourier transform (FFT). The focus of this

72 paper is on how to compute displacement time histories (blue curve).

73

74

5

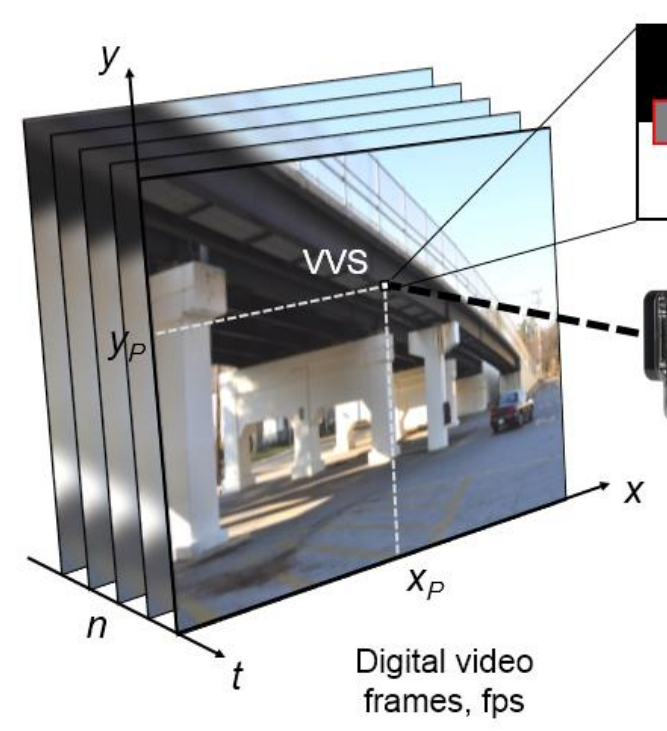

Digital video
frames, fps

4

.

Figure 1. Illustration of the proposed methodology to measure structural vibrations using Eulerian-based virtual visual sensors (VVS). Acronyms: BWT = black-and-white target, FFT = Fast Fourier transform, IDT = intensity-to-displacement transform.

\section{Background and Theory}

As we discussed in [11], [12], change of intensity observed by our Eulerian-based virtual visual sensor (VVS) did previously not directly correspond to a physical quantity such as displacement, velocity, or acceleration. In this study, we evaluate the use of simple printed black-and-white targets (BWT) that allow the measurement of actual dynamic displacements, as visualized in Fig.

2. For an ideal BWT target observed in a digital video with dimensions $L_{t} \mathrm{x} W_{t}$ (pixel x pixel), the 
85 pattern colors are represented by the minimum and maximum intensity values (Imin. and $I_{\max }$,

86 respectively) corresponding to 0 (= black) and 255 (= white), respectively. The displacement varies

87 linearly with VVS patch intensity, $I_{p}(t)$ :

$$
I_{p}(t)=\frac{\left(I_{\max }-I_{\min }\right)}{L_{p}} x(t)+n_{p}(t)
$$

91 where $I_{p}(t)$ is the average pixel intensity across the patch area, $A_{p}=W_{p} \mathrm{x} L_{p}$ (pixel x pixel) for a

92 frame at time instant, $t(\mathrm{sec}), x(t)$ (pixel) measured displacement, and $L_{p}$ (pixel) is the length of the

93 patch. It is assumed that intensity across the width of the patch, $W_{p}$ (pixel) is constant and it

94 therefore does not appear in Eq. (1). It should be noted that the patch length, $L_{p}$ (pixel) should be

95 large enough to account for the maximum displacement amplitude, $A$ (pixel), i.e. $L_{p}>A$. At the

96 same time, the length of the target, $L_{t}$ (pixel) needs to be able to accommodate for the patch length,

$97 L_{p}$ (pixel), i.e. the target cannot leave the patch, otherwise the relationship becomes non-linear.

98 Finally, there is no perfect BWT target (with perfect black $(I=0)$ or white $(I=255)$ intensity

99 values) in a real setting and measurement noise is always present. The total average noise of the

100 patch, $n_{p}(t)$ can be defined as:

101

102

$$
n_{p}(t)=\frac{1}{N} \sum_{i=1}^{N} n_{i}(t)
$$

104 where $N$ is the total number of pixels in the VVS patch and $n_{i}$ is the noise present in pixel $i$. 


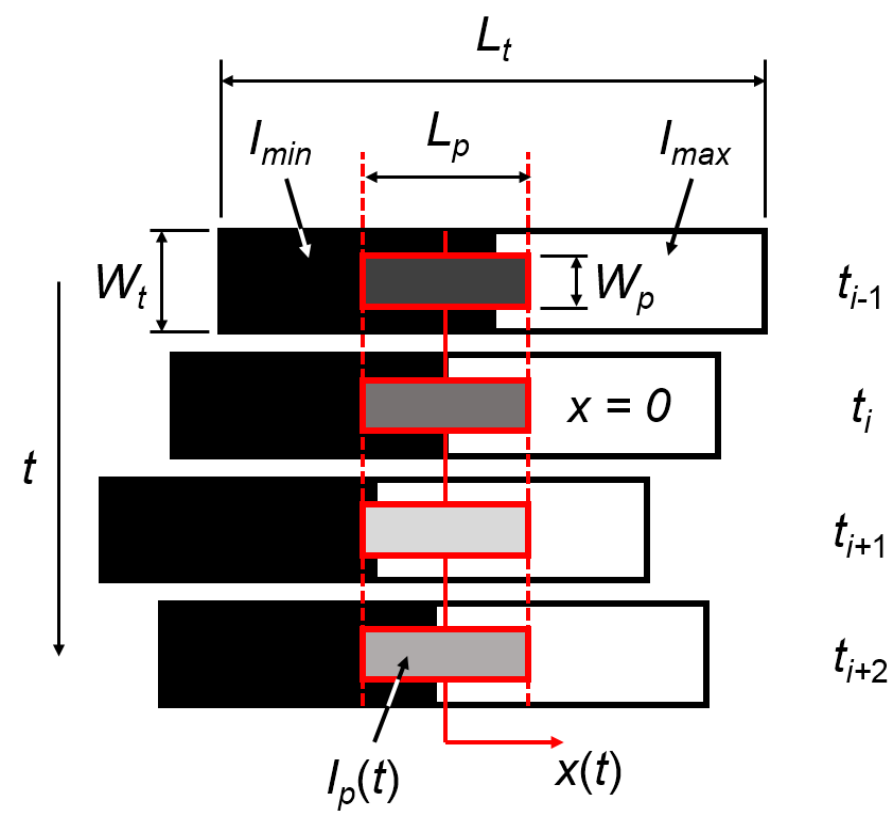

107 Figure 2. Illustration of the VVS measurement process using a black-and-white target (BWT):

108 the target, which is attached to the vibrating structural element, moves in the $x$-direction relative 109 to a fixed patch of pixels, i.e. having Eulerian-coordinates, as a function of time, $t$.

111 As an alternative to Eq. (1), VVS patch intensity, $I_{p}(t)$ can also be computed as the average 112 intensity value of all pixels across the patch area, $A_{p}$ :

$$
I_{p}(t)=\frac{1}{N} \sum_{i=1}^{N} I_{i}(t)
$$

116 where $N$ is the total number of pixels in the VVS patch and $I_{i}$ is the intensity value of pixel $i$. 
118

In order to correlate the observed intensity values to actual displacement, a calibration constant, $B(\mathrm{~mm} / \mathrm{pixel})$ needs to be determined. This is done by dividing the actual length of the BWT (mm) by the corresponding number of pixels (pixels) observed from a selected frame of the video. For the case where the camera is not oriented perpendicular to the displacement component of interest, i.e. when $b \neq 0$, a geometric correction factor, $C$ (unitless) applies. This factor is calculated based on the location of the camera $(O)$ and the center location of the target $(T)$, as illustrated in Fig. 3:

$$
C=\frac{1}{\cos \left[\tan ^{-1}\left(\frac{b}{a}\right)\right]}
$$

133 Motion observed at angles $\neq 90$ Degrees about the axis aligned with that motion (in our case 134 vertical) are not affected in any significant manner and are thus not considered. 
135 Considering that $I_{p}(t)$ is known and by using the calibration constant, $B(\mathrm{~mm} / \mathrm{pixel})$ and the

136 geometric correction factor, $C$, the actual dynamic displacement of the target, $u_{a c t}(t)$ can be 137 computed using the following relationship:

$$
u_{\text {act }}(t)=B \cdot C \cdot I_{p}(t)(\mathrm{mm})
$$

141 Changing lighting conditions affect the measured intensity values and thus introduce an error in

142 the prediction of $u_{a c t}(t)$. This is really only a problem for long-term measurements (e.g. for SHM

143 applications) but can be addressed by continuously normalizing the difference of the measured 144 intensity values on the target $\left(I_{\max }-I_{\min }\right)$ when lighting conditions change. For short-term 145 measurements (e.g. annual impact or load tests) where the test time can be selected accordingly, 146 this effect is negligible.

148 The presence of noise may require implementation of a noise reduction technique. Fortunately, 149 for a BWT the averaging process (expressed by Eqs. (1) and (3)) by itself helps reducing the noise, 150 i.e. the power of the noise reduces directly with the number of pixels in the patch, $N$. Assuming 151 that the noise is independent of the signal and can be represented by a stationary process, we arrive 152 at:

153

$$
\sigma^{2}\left(I_{p}\right)=\frac{\left(I_{\max }-I_{\min }\right)^{2}}{L_{p}^{2}} \sigma^{2}(x)+\frac{\sigma^{2}\left(n_{p}\right)}{N}
$$




\section{3}

Eq. (6) relates the size of the VVS patch and the power of the noise and the signal. As can be seen from the second term of the right hand side of Eq. (6), as the number of pixels increases the power of the noise decreases. However, increasing the length of the patch will have the same effect on the power of the signal, hence keeping the length as short as possible and the width as large as possible will maximize the $S N R$. Substituting $N$ for $W_{p} L_{p}$ in Eq. (6), which is width $\mathrm{x}$ length of the patch, we can get the following expression for the signal-to-noise ratio (SNR):

$$
S N R=\frac{\frac{\left(I_{\max }-I_{\min }\right)^{2}}{L_{p}^{2}} \sigma^{2}(x)}{\frac{\sigma^{2}\left(n_{p}\right)}{W_{p} L_{p}}}=\frac{W_{p}}{L_{p}}\left(I_{\max }-I_{\min }\right)^{2} \frac{\sigma^{2}(x)}{\sigma^{2}\left(n_{p}\right)}
$$

Eq. (7) shows that in order to reach the maximum $S N R$ one has to maximize the $W_{p}$-to- $L_{p}$ ratio keeping in mind that $L_{p}$ should be able to cover the maximum displacement amplitude, $A$, as discussed earlier. For a specific camera and lighting conditions, the pixel noise power can be assumed constant and the appropriate size of the patch can be specified based on the desired SNR.

The other factor that should be discussed in Eq. (7) is the second factor, $\left(I_{\max }-I_{\min }\right)$, which has a more significant effect on the $S N R$. It can be concluded from Eq. (7) that the higher the contrast between black and white in the target, the higher the SNR will be.

\section{Experiments}

\subsection{Laboratory Setup and Instrumentation}

A laboratory-scale three-degree-of-freedom structural system (Total height $=610 \mathrm{~mm}(2 \mathrm{ft})$ as shown in Fig. 4 (d) was used to evaluate the accuracy of the methodology proposed in Section 2. 
177 The structure was excited by introducing random initial displacements at two locations on the

178 structure by hand followed by a sudden release to initiate free vibration. A digital camera (GoPro

179 Hero 3, shown in Fig. 4 (c))) capturing the free vibration response was located $305 \mathrm{~mm}(1 \mathrm{ft})$ away

180 from the structure. The displacement of the first floor was also measured using a $12.7 \mathrm{~mm}(0.5 \mathrm{in})$

181 amplitude potentiometer (Fig. 4 (b)) connected to a high-speed data acquisition system (Fig. 4 (a))

182 using a sampling frequency of $1200 \mathrm{~Hz}$. The frame rate of the digital camera was 60 frames per 183 seconds (fps).

184

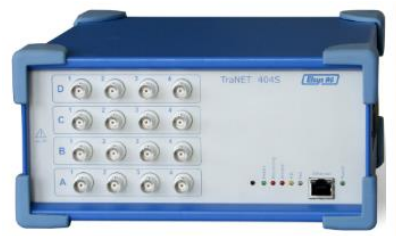

(a)

(b)
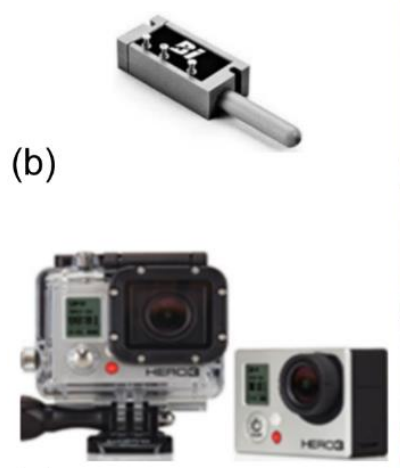

(c)

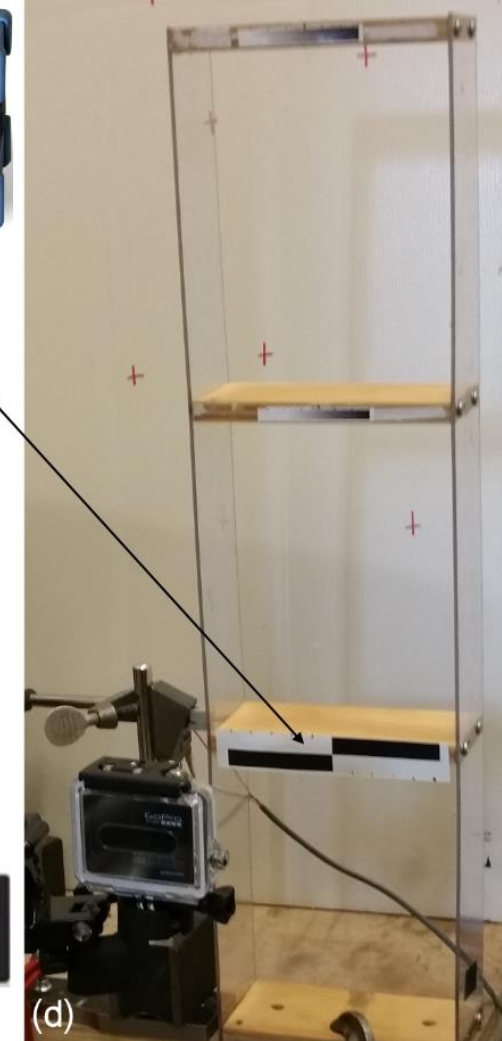

(d)

186 Figure 4. Experimental setup: (a) high-speed data acquisition system, (b) potentiometer to measure displacements at the first story mass, (c) digital camera to collect VVS data, and (d) three-degree-of-freedom laboratory structure. 
191 In order to compare the two measured signals, two steps have to be taken: (1) synchronization of

192 the signals in the time domain and (2) multiplication of the measured signals by their appropriate

193 calibration factors to obtain actual displacement from the measured data. It is good to mention that

194 the $\left(I_{\max }-I_{\min }\right)$ value was assumed to be constant, which proved to be a correct assumption based

195 on the data. Also the calculation of this value was based on the average of a black and white patch

196 of pixels on the target that was taken from a snapshot of the videos. For step (1), both camera and

197 potentiometer data were interpolated linearly to two equivalent $3000 \mathrm{~Hz}$ signals. Based on the

198 maximum correlation between the two signals, the time lag between the two signals was calculated

199 and one of the signals shifted so that they had a common time axis. In order to achieve actual

200 displacement for step (2), the potentiometer was calibrated against a precision height gage. The

201 mean calibration factor was found to be $1.257 \mathrm{~mm} / \mathrm{V}(0.0495 \mathrm{in} / \mathrm{V})$. For the camera intensity data,

202 the known target length, $L_{t}$ was measured in a video frame in terms of pixels, which produced a 203 mean calibration factor of $0.279 \mathrm{~mm} / \mathrm{pixel}(0.011 \mathrm{in} / \mathrm{pixel})$.

205 In addition to the independent application of calibration factors as described above, it was also 206 possible to multiply the VVS intensity data by a factor that minimizes the second norm of 207 difference, or error, between the two measurements. This case represents the optimal estimate of 208 the displacement for the VVS, assuming the potentiometer represents an accurate reference 209 measurement. Obviously, in a real life scenario only the first approach can be used where a 210 calibration factor has to be estimated from the video data. It should be noted that the potentiometer 211 serves as the reference measurement but does not necessarily produce a more accurate 212 displacement. This was particularly visible at the peak displacement points and is discussed in 213 more detail in Section 4.1. 


\section{Results}

\section{$216 \quad 4.1$ Accuracy of Proposed Approach}

217 Two VVS patch sizes, $W_{p} \times L_{p}=40 \times 50$ and $40 \times 100$ pixels, were selected to study how the 218 accuracy of the measurements change with the size of the VVS patch, $A_{p}$. Fig. 5 shows a snapshot 219 of a video frame with the target and the two evaluated VVS patch sizes.

(a)

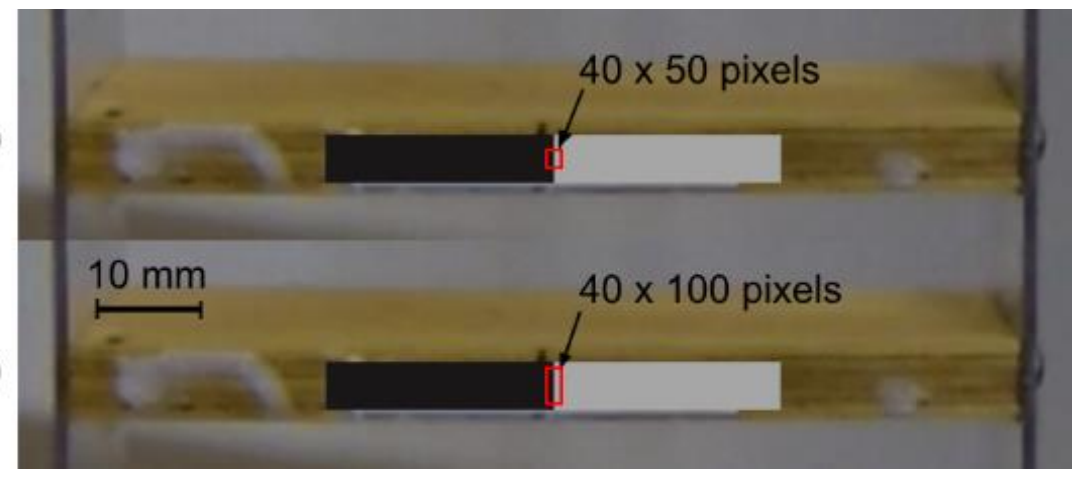

Figure 5. Photo of BWT target with two VVS patch sizes: (a) $40 \times 50$ pixel patch and (b) $40 \times$ 100 pixel patch.

226 described in Section 3.2. As can be observed from Fig. 6 (a), the displacements measured by the

227 VVS and the potentiometer are, qualitatively, in close agreement for both patch sizes. However, 228 the inserts in Fig. 6 (a) reveal that the end of the signal of the 40 x 100-pixel patch resembles the 229 potentiometer's measurement more closely. A direct correlation between the two measurements 230 (Fig. 6 (b)) shows approximately a straight line with a slope of 0.95 and 0.92 with a squared 231 correlation coefficient of 99.6 and $99.9 \%$ for the patch size of $40 \times 50$ and $40 \times 100$ pixels, 232 respectively. The absolute prediction error at the $95 \%$ confidence level was determined by 
233 measuring the distance between the 95\% prediction limits shown in Fig. 6 (b) (red dotted lines)

234 and found to be 0.12 and $0.24 \mathrm{~mm}(0.0047$ and 0.0094 in) for the patch size of $40 \times 50$ and $40 \mathrm{x}$

235100 pixels, respectively. Furthermore, in Fig. 6 (c), which shows the absolute value of the

236 difference between the two measurements, less than $2 \%$ of the signal difference is greater than a

237 pixel size and roughly $90 \%$ of the time the difference is less than half of a pixel size. It can further

238 be observed that the difference shows distinct evenly-spaced peaks that are highest at the beginning

239 of the signal. Also, they appear to coincide with the peak amplitudes of the signal. The difference

240 is likely due to an error in the potentiometer measurement, when the direction of the displacement

241 changes. Unfortunately, it was not possible for us to ascertain this claim completely. In the future,

242 we plan to perform further laboratory tests using a laser vibrometer. Despite this uncertainty, our

243 data shows that subpixel-level accuracy is achievable with high confidence. The actual difference

244 in terms of noise can be observed by visually comparing the curves in Fig. 6 (c) between 4 and 6

245 s. The distribution of the error with mean, $\mu$ and standard deviation, $\sigma$ is shown in Fig. 6 (d). It can

246 be observed from the distribution of the signal difference that it appears to follow a Normal

247 distribution, as assumed in Section 2. 
a)
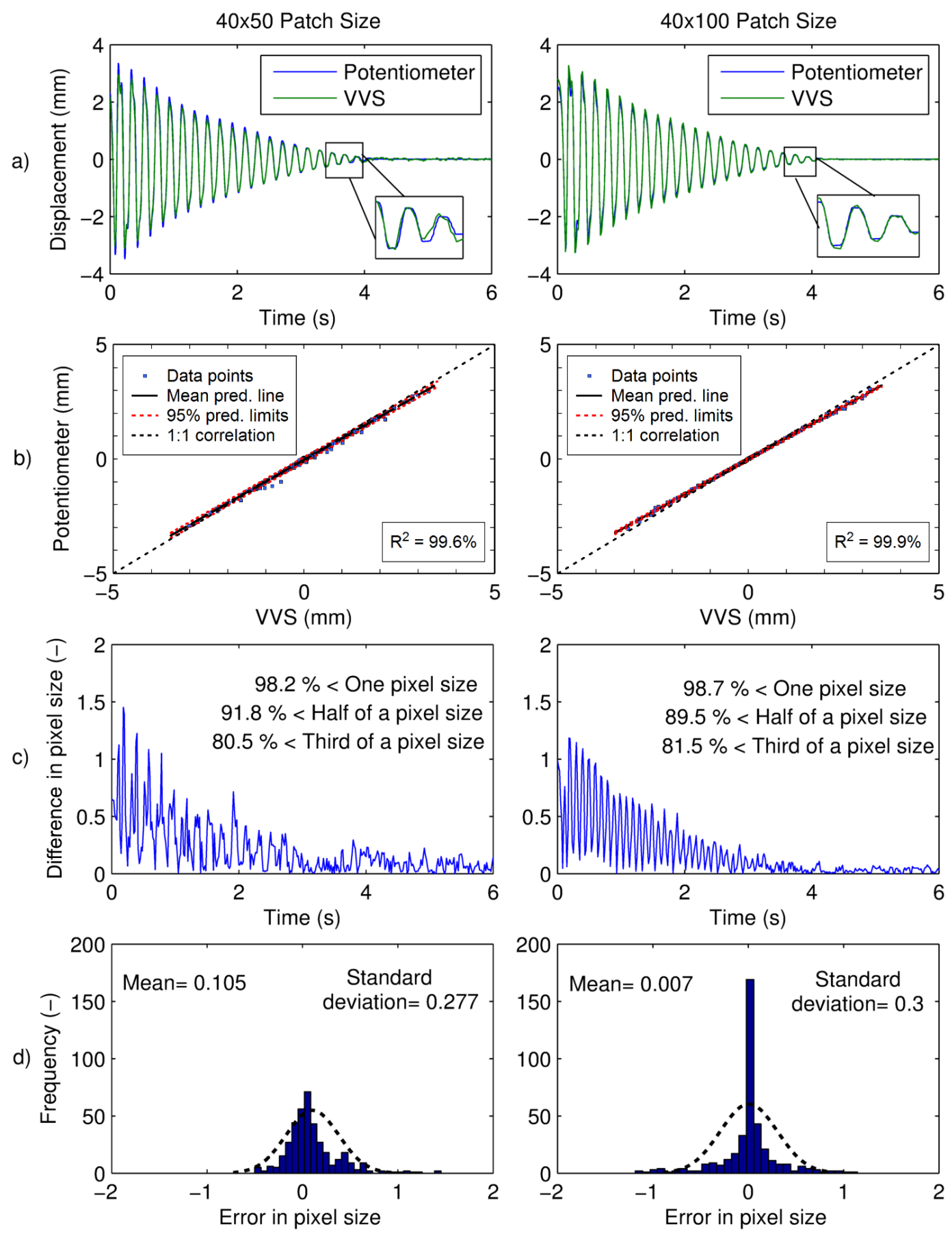

Figure 6. Comparison of results using independent calibration factors, $40 \times 50$ pixels (left column) and $40 \times 100$ pixels (right column): (a) Time history signals of VVS patch data and

251 potentiometer, (b) correlation between the two measurements with regression lines, (c) absolute 252 value of the difference between the two signals (errors), and (d) histogram of the errors. 
Fig. 7 shows the case where the calibration factor for the VVS was optimized as discussed in

255 Section 3.2. Fig. 7 (a) compares with Fig. 6 (a) while correlation plots shown in Fig. 7 (b) are even

256 better compared to Fig. 6 (b). The slope of the prediction line in Fig. 6 (b) is 0.99 and 0.98 with a

257 squared correlation coefficient of 99.5 and $99.9 \%$ for the patch size of $40 \times 50$ and $40 \times 100$ pixels,

258 respectively. The absolute prediction error at the $95 \%$ confidence level, computed as described

259 earlier, was found to be 0.26 and $0.11 \mathrm{~mm}(0.01$ and $0.0043 \mathrm{in})$ for the patch size of $40 \times 50$ and

26040 x 100 pixels, respectively. The maximum signal difference (Fig. 7 (c)) is reduced by almost

261 half of a pixel size as compared to Fig. 6 (c). Comparing the patch sizes in Fig. 7 (d), it can be

262 observed that the standard deviation of the pixel error has been significantly decreased from 0.23

263 pixels to 0.12 pixels for the $40 \times 50$-pixel patch compared to the $40 \times 100$-pixel patch, respectively.

264 Also, with a confidence of more than $90 \%$, the error in the smaller patch is less than one third of a

265 pixel size while in the bigger patch it is less than one fifth of a pixel size. Again, this approach

266 represents the case where the calibration factor for the VVS sensor was optimized by minimizing

267 the difference between the two measurements.

268 In conclusion from Figs. 6 and 7, we have demonstrated that subpixel accuracy can be achieved

269 with high confidence, even without implementing a computationally-expensive block matching

270 algorithm, and that estimates of the dynamic displacement can be achieved in a laboratory setting

271 with absolute prediction errors of approximately $0.25 \mathrm{~mm}(0.01 \mathrm{in})$. 
a)
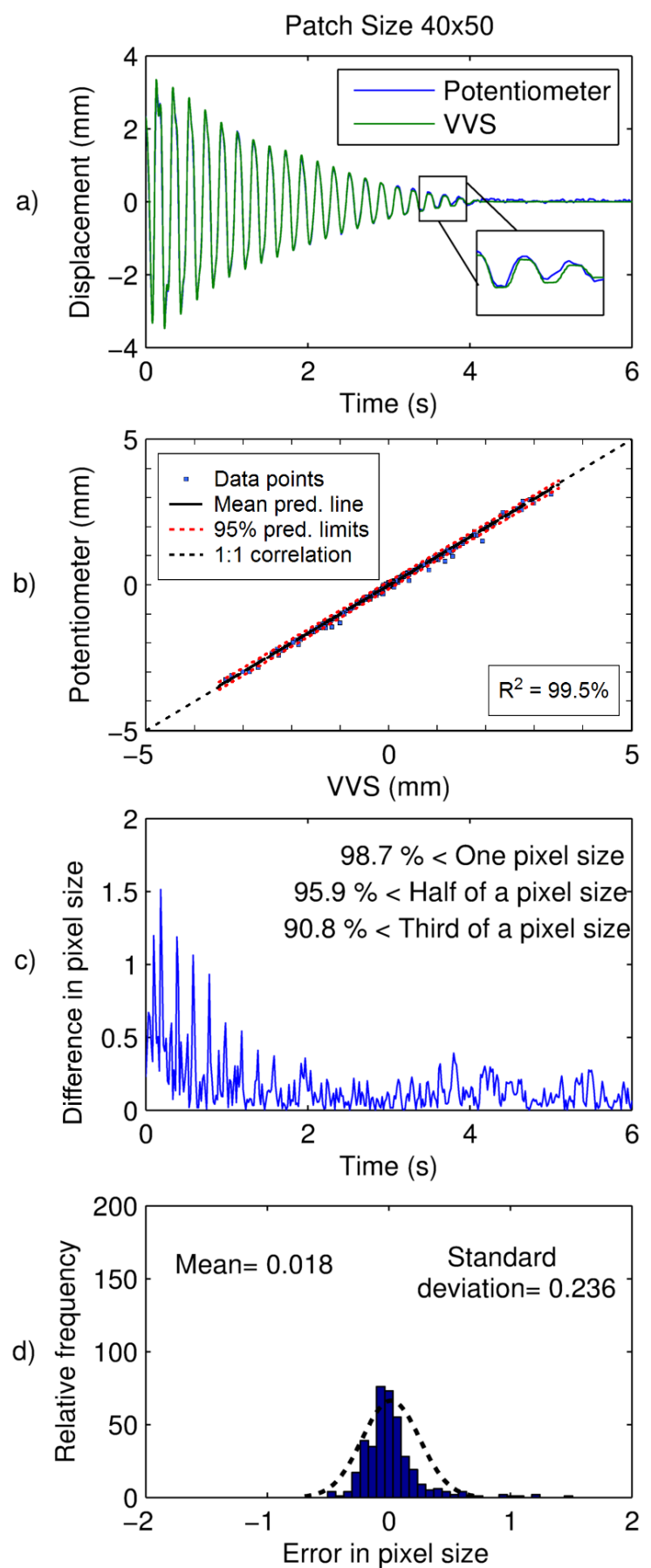

Patch Size $40 \times 100$
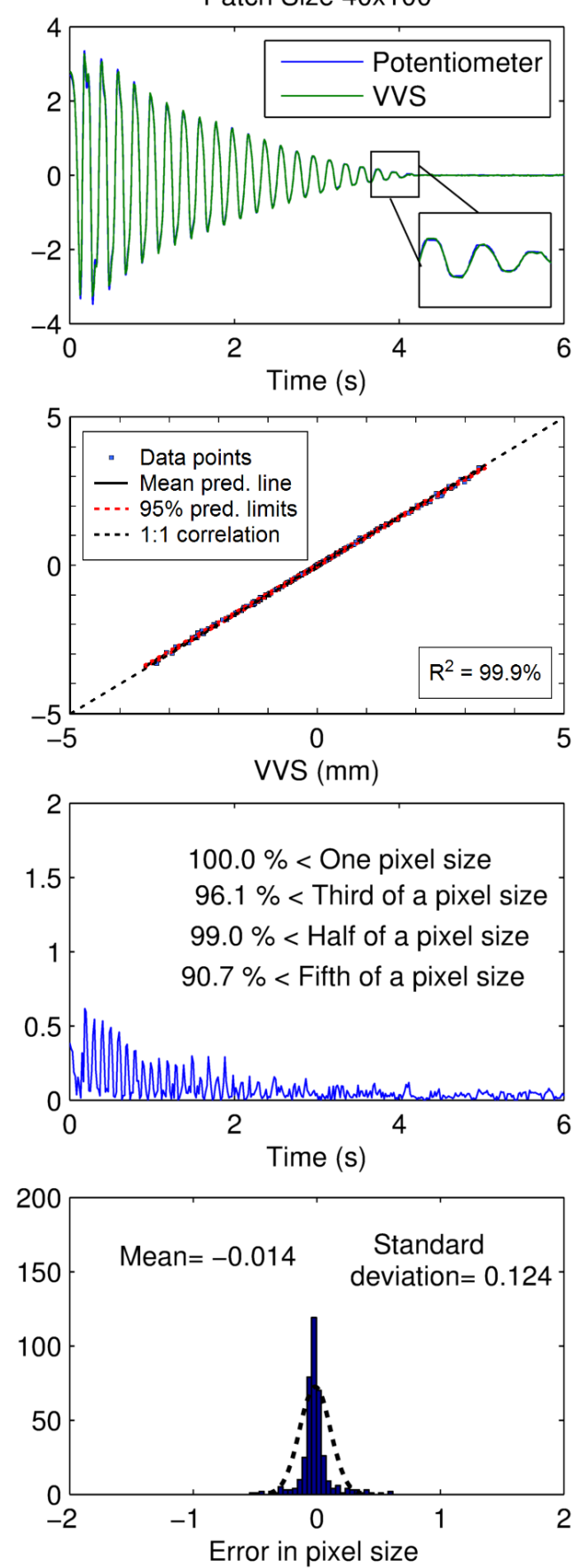

Figure 7. Comparison of results using calibration factors based on minimized difference

275 between measurements, $40 \times 50$ pixels (left column) and $40 \times 100$ pixels (right column): (a)

Time history signals of camera and potentiometer, (b) correlation between the two 
Table 1 summarizes the main results of the accuracy evaluation presented in this section. It can 281 be seen that the larger VVS patch ( $40 \times 100$ pixels) was closer to the potentiometer reading 282 compared to the smaller patch $(40 \times 50$ pixels $)$. For the displacements computed using the 283 independent factors, the larger sized VVS patch was closer to the potentiometer measurement. 284 However, the standard deviation of the difference for the larger patch remained the same because 285 of the calibration issues explained earlier. While this type of comparison can likely not be 286 performed in the field, as it would require an independent physical measurement of the 287 displacement, it allowed us to isolate and study the calibration errors and the inherent irreducible 288 noise using the proposed VVS.

\section{$290 \quad 4.2$ Relationship of Noise and Patch Size}

291 Fig. 8 (d) shows the relationship between patch noise and number of pixels in a VVS patch as 292 defined theoretically by Eq. (7) and observed experimentally. As can be seen in Fig. 8 (a), the 293 power of the noise is close to the theoretical values. Figs. 8 (b) and (c) show the distribution of the 294 noise for one pixel and a patch of $10 \times 10$ pixels. As can be observed, the noise in the patch follows 295 a Normal distribution and its power is one order of magnitude smaller than that for one pixel. Also, 296 the SNR values approximately change linearly with the width to length ratio of the patch as 297 predicted from Eq. (7). This validates the theoretical framework presented in Section 2. 


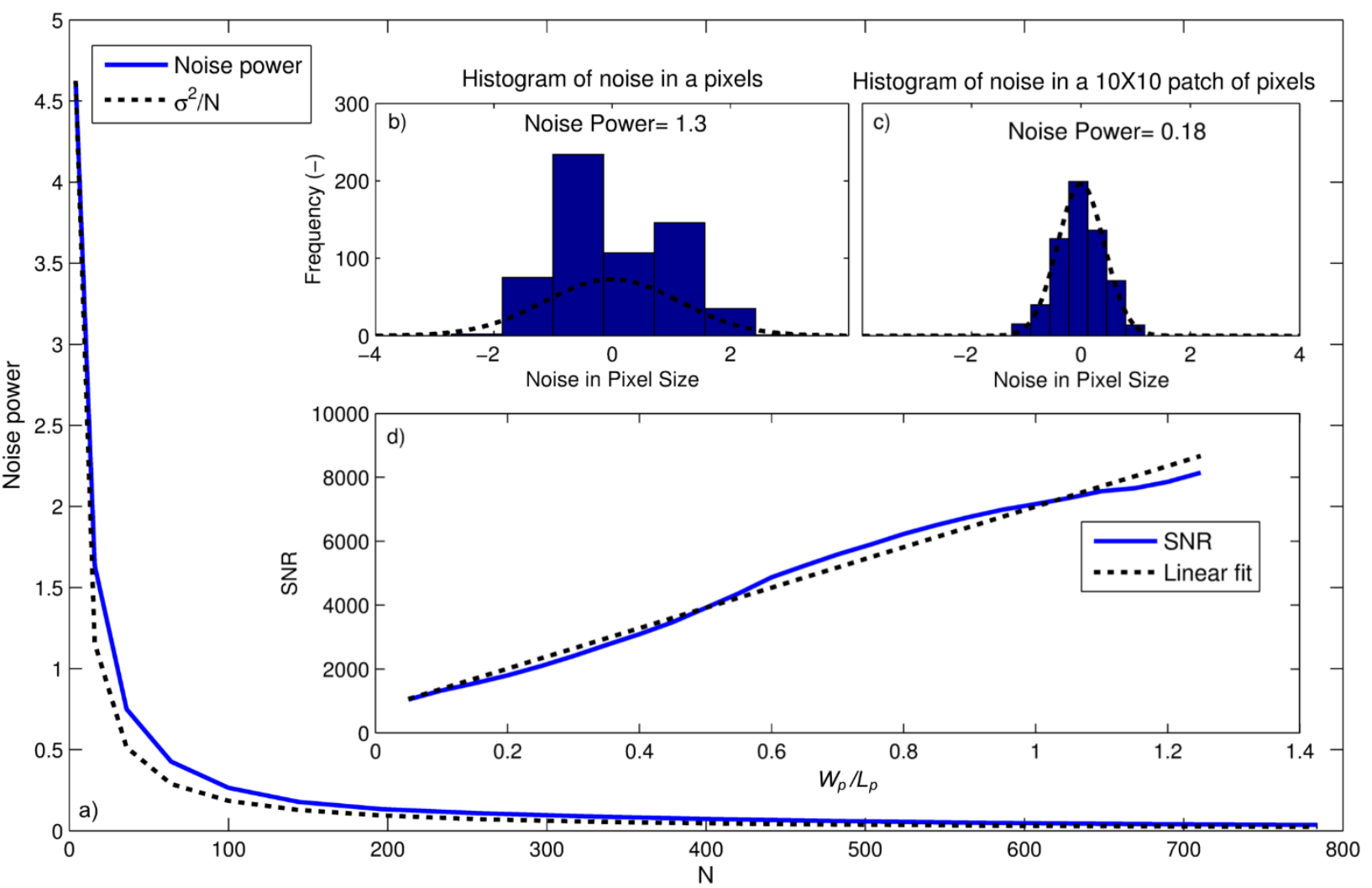

Figure 8. Noise power and the signal-to-noise ratio (SNR): (a) Power of the noise vs. the number of pixels in the VVS patch $(M)$, (b) histogram of noise in one pixel, (c) histogram of noise in a $10 \times 10$-pixel patch, and (d) the SNR values vs. width over length of the patch.

\subsection{Dynamic In-Service Load Test on the Streicker Bridge}

305 In order to evaluate the applicability of the proposed methodology in a real-world scenario, the

306 Streicker Bridge was tested dynamically with a black-white target (BWT) to compute

307 displacement. Located on the Princeton University campus, the bridge has a unique design with a

308 straight main deck section supported by a steel truss system underneath and four curved ramps

309 leading up to the straight sections, as shown in Fig. 9. One of the ramps was instrumented with a

310 fiber-optic measurement system during construction by Br. Branko Glisic from Princeton

311 University [14]. For our test, we installed an off-the-shelf Canon EOS Rebel T4i camera with a 
312 standard Canon EF 75-30mm zoom lens aimed at one of the ramps, to take a 60 fps video while a

313 number of volunteers jumped up and down on it. A VVS patch having $60 \times 20$ pixels was chosen

314 to compute displacements. As can be seen in Fig. 8, the VVS is located at a black-and-white edge

315 on a target mounted to the edge of the bridge slab. This target was set up by Dr. Maria Feng's

316 research team from Columbia University, who collected data for evaluation of their own video-

317 based monitoring methodology [15]. The relationships in Eqs. (1) and (5) were used to compute

318 the actual vertical dynamic displacement from the collected VVS patch. The calibration constant,

$319 B$ was estimated from the target size as $17.3(\mathrm{~mm} / \mathrm{pixel})$; the geometric correction factor, $C$

320 estimated to be 1.02 .

321 


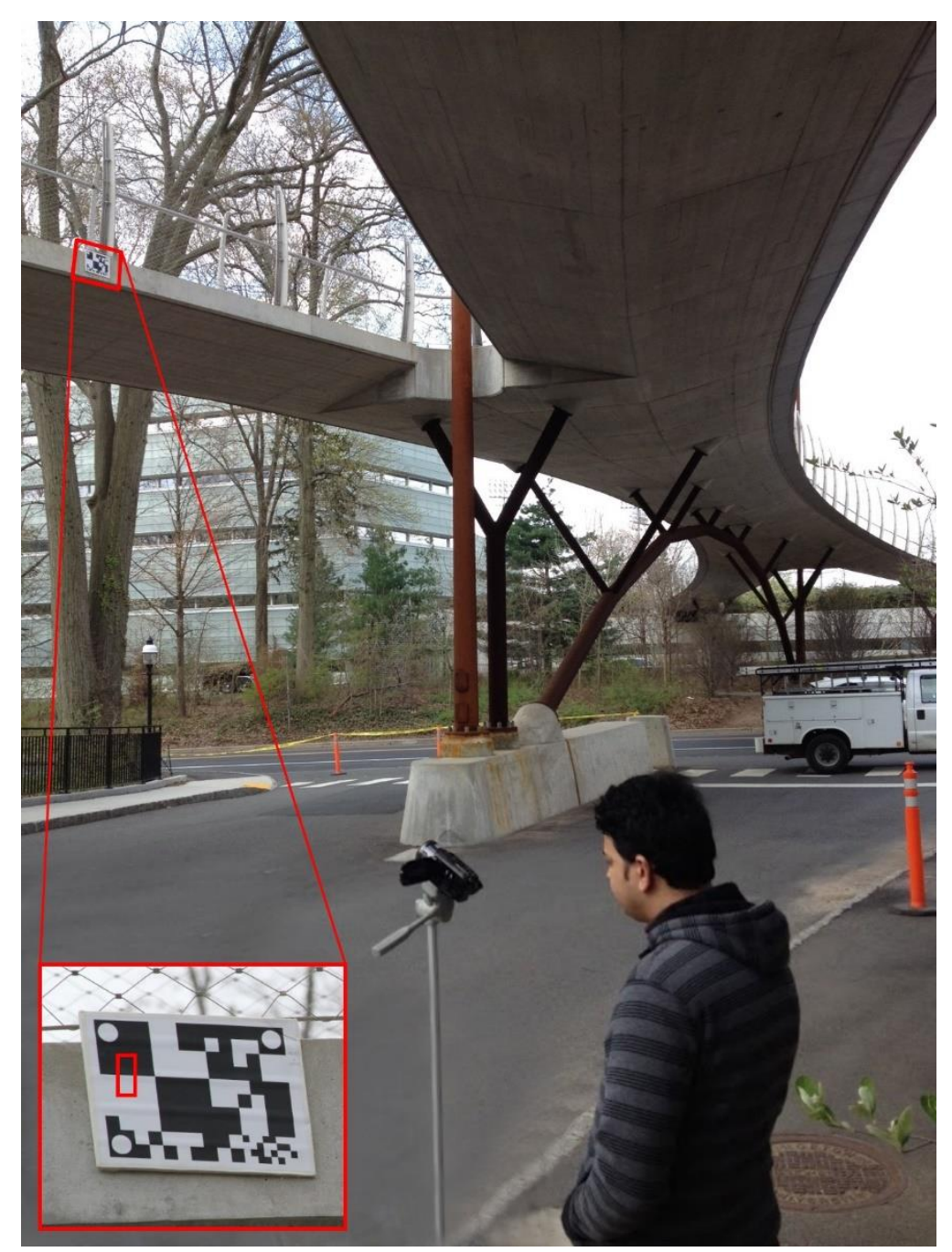

323 Figure 9. Photograph of the Streicker Bridge showing the measurement setup and the location

324 of the VVS. The insert shows the location of the $60 \times 20$ pixel VVS patch (red rectangular). The target was installed by Dr. Maria Feng's research team from Columbia University [15].

327 The computed vertical displacement response of the ramp section due to the described dynamic

328 forcing for a duration of 15 seconds is presented in Fig. 10. In our earlier paper we already reported

329 that the natural frequencies for this same test were found to be the same as those measured by the

330 fiber-optic measurement system [14]. Although we have no other physical measurement available

331 to directly compare and verify our computed displacement, it is comparable in amplitude to what 
332 the Columbia University team reported [15]. Also, the frequency peak is exactly the same as 333 reported by the same group.
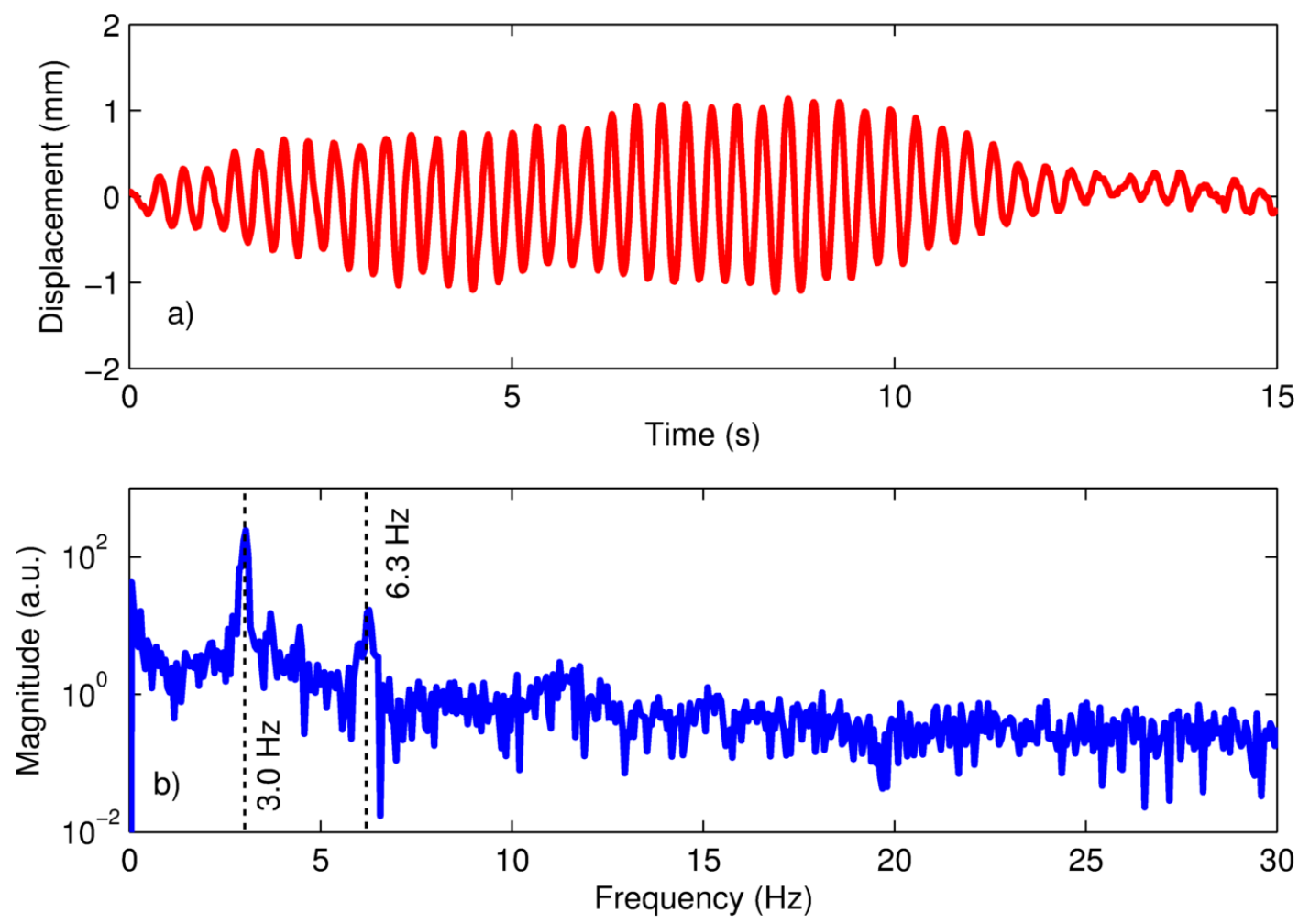

Figure 10. Results from the dynamic load test on the Streicker Bridge: (a) Computed actual

\section{Discussion and Conclusions}

340 The objective of this study was to evaluate the possibility of computing actual dynamic

341 displacements using Eulerian-based virtual visual sensors (VVS). This is based on the idea that

342 either an edge of a vibrating structural element or a black-and-white target (BWT) can be

343 monitored by a patch of pixels. The noise in the VVS sensor was found to be inversely related to

344 the patch size. The following conclusions can be made from on our study: 
345 - The use of BWT allows for accurate computation of dynamic displacements of a vibrating

346 structural element comparable to the measurements from a potentiometer.

347 - The laboratory tests demonstrated that sub-pixel accuracy can be achieved similar to block

348 matching algorithms. The absolute prediction error at the $95 \%$ confidence limit was found to

349 be approximately $0.25 \mathrm{~mm}(0.01 \mathrm{in})$ relative to the reference measurement.

350 - The accuracy in the measurement of the displacement implies that change of intensity is highly

351 sensitive to even tiny amounts of movement, which results in the fact that natural frequencies

352 can be measured as proposed in [11]-[13] even if the displacement is much less than a pixel

353 size.

354 - Our proposed approach also works in the field, as demonstrated by the measurements of the

355 Streicker Bridge. A direct validation was not possible since no other physical displacement

356 data was available, which is typically the case for field measurements. However, the frequency

357 content of the signal has already been verified in [18] and the displacement amplitude as well

358 as the frequency peak is comparable to what the team from Columbia University found [15].

359 - The influence of camera movement and changes in lighting conditions need to be addressed

360 further in future research.

362 Acknowledgments

363 The support by a Center for Advanced Infrastructure and Transportation University Transportation

364 Research (CAIT-UTC) grant (Contract No. DTRT12-G-UTC16) and the Department of Civil and

365 Environmental Engineering at the University of Delaware for this study is greatly appreciated. We

366 further thank our colleague Prof. Branko Glisic, who facilitated access for the field test on the

367 Streicker Bridge located on Princeton University's campus. Finally, we would like to thank Dr. 
368 Nakul Ramanna for assisting with the field test, Dr. Maria Feng and her team from Columbia

369 University for allowing us to use their target, and Mr. Marcus Schwing for his expertise in photo 370 editing.

372 References

373 [1] A. Deraemaeker and K. Worden, New trends in vibration based structural health monitoring. 2012.

375 [2] S. W. S. Doebling, C. R. C. Farrar, M. B. M. Prime, and D. W. D. Shevitz, "Damage identification and health monitoring of structural and mechanical systems from changes in their vibration characteristics: a literature review," 1996.

C. R. Farrar and K. Worden, "An introduction to structural health monitoring.," Philos. Trans. A. Math. Phys. Eng. Sci., vol. 365, no. 1851, pp. 303-315, 2007.

H. H. Nassif, M. Gindy, and J. Davis, "Comparison of laser Doppler vibrometer with contact sensors for monitoring bridge deflection and vibration," NDT E Int., vol. 38, no. 3, pp. 213218, Apr. 2005.

B. D. Lucas and T. Kanade, "An Iterative Image Registration Technique with an Application to Stereo Vision," IJCAI, vol. 130, pp. 674-679, 1981.

[6] H. Leclerc, J. Périé, S. Roux, and F. Hild, "Integrated digital image correlation for the identification of mechanical properties," Comput. Vision/Computer Graph. ..., pp. 161171, 2009.

R. C. Oats, D. K. Harris, T. (Tess) M. Ahlborn, and H. A. de Melo e Silva, "Evaluation of the Digital Image Correlation Method as a Structural Damage Assessment and Management Tool," in Transportation Research Board 92nd Annual Meeting, 2013. 
391 [8] I. B. Mohammad Bolhassani, Satish Rajaram, Ahmad A. Hamid, Antonios Kontsos, "Damage detection of concrete masonry structures by enhancing deformation measurement using DIC," in Nondestructive Characterization and Monitoring of Advanced Materials, Aerospace, and Civil Infrastructure X, SPIE, 2016.

[10] Y.-Z. Song, C. R. Bowen, A. H. Kim, A. Nassehi, J. Padget, and N. Gathercole, "Virtual visual sensors and their application in structural health monitoring," Struct. Heal. Monit., vol. 13, no. 3, pp. 251-264, Feb. 2014.

[11] T. Schumacher and A. Shariati, "Monitoring of structures and mechanical systems using virtual visual sensors for video analysis: fundamental concept and proof of feasibility.," Sensors (Basel)., vol. 13, no. 12, pp. 16551-64, Jan. 2013.

[12] A. Shariati, T. Schumacher, and N. Ramanna, "Eulerian-based virtual visual sensors to detect natural frequencies of structures," J. Civ. Struct. Heal. Monit., vol. 5, no. 4, pp. 457468, 2015.

[13] A. Shariati and T. Schumacher, "Oversampling in virtual visual sensors as a means to recover higher modes of vibration," in AIP Conference Proceedings (Proceedings of QNDE 2014, July 20-25, Boise, ID.), no. Dic, pp. 1-7.

[14] B. Glisic, J. Chen, and D. Hubbell, "Streicker Bridge: a comparison between Bragg-grating long-gauge strain and temperature sensors and Brillouin scattering-based distributed strain and temperature sensors," in SPIE Smart Structures and Materials + Nondestructive Evaluation and Health Monitoring, 2011, p. 79812C-79812C-10. 
414 [15] D. Feng, M. Q. Feng, E. Ozer, and Y. Fukuda, "A Vision-Based Sensor for Noncontact 415 Structural Displacement Measurement.," Sensors (Basel)., vol. 15, no. 7, pp. 16557-75, Jan. 2015.

417

418 
Table1. Summary table of accuracy evaluation.

\begin{tabular}{|c|c|c|c|c|}
\cline { 2 - 4 } \multicolumn{1}{c|}{} & \multicolumn{2}{c|}{$\begin{array}{c}\text { Independent calibration } \\
\text { factors } \\
\text { (see Fig. 6) }\end{array}$} & \multicolumn{2}{c|}{$\begin{array}{c}\text { Minimization of signal } \\
\text { difference } \\
\text { (see Fig. 7) }\end{array}$} \\
\hline Size of the patch & $40 \times 50$ & $40 \times 100$ & $40 \times 50$ & $40 \times 100$ \\
\hline Correlation Coefficient & 0.998 & 1.000 & 0.998 & 1.000 \\
\hline Maximum difference in pixel size & 1.5 & 1.2 & 1.5 & 0.6 \\
\hline Mean of the difference & 0.105 & 0.007 & 0.018 & -0.014 \\
\hline Standard deviation of the difference & 0.277 & 0.300 & 0.236 & 0.124 \\
\hline
\end{tabular}

420 